\title{
Posture, Head Stability, and Orientation Recovery During Vestibular Regeneration In Pigeons
}

\author{
J. David Dickman And Insook Lim
}

Department of Anatomy and Neurobiology, Washington University, St. Louis, MO 63110, USA

Received: 21 November 2003; Accepted: 13 April 2004; Online publication: 12 August 2004

\section{ABSTRACT}

Compensatory behavior such as oculomotor, gaze, and postural responses that occur during movement largely depend upon a functioning vestibular system. In the present study, the initial loss and subsequent recovery of postural and head stability in pigeons undergoing vestibular regeneration were examined. Adult pigeons were trained to manipulate a straight run chamber to peck an illuminated key for fluid reward. Six behavioral measures assessing performance, posture, and head stability were quantified. These included run latency, steps (walking), path negotiation (lane changes), gaze saccades, head bobs, and head shakes. Once normative values were obtained for four birds, complete lesion of all receptor cells and denervation of the epithelia in the vestibular endorgans were produced using a single intralabyrinthine application of streptomycin sulfate. Each bird was then tested at specific times during regeneration and the same behavioral measures examined. At 7 days post-streptomycin treatment (PST), all birds exhibited severe postural and head instability, with tremors, head shakes, staggering, and circling predominating. No normal trial runs, walking, gaze saccades, or head bobs were present. Many of these dysfunctions persisted through 3-4 weeks PST. Gradually, tremor and head shakes diminished and were replaced with an increasing number of normal head bobs during steps and gaze saccades. Beginning at 4 weeks PST, but largely inaccurate, was the observed initiation of directed steps, less staggering, and

Correspondence to: J. David Dickman • Department of Otolaryngology -Washington University • 660 South Euclid • Box $8115 \cdot$ St. Louis, MO 63110. Telephone: (314) 747-7221; fax: (314) 747-7206; email: ddickman@wustl.edu some successful path negotiation. As regeneration progressed, spatial orientation and navigation ability increased and, by 49 days PST, most trials were successful. By 70 days PST, all birds had recovered to pretreatment levels. Thus, it was observed that ataxia must subside, coincident with normalized head and postural stability prior to the recovery of spatial orientation and path navigation recovery. Parallels in recovery were drawn to hair cell regeneration and afferent responsiveness, as inferred from present results and those in other investigations.

Keywords: vestibular, otolith, gaze, ototoxicity, regeneration, motor-learning, spatial orientation

\section{INTRODUCTION}

Birds must stabilize their vision during head motion as experienced in walking, running, or flight behavior. When the head is free to move, visual stability is accomplished through gaze control, a postural neuromotor behavior composed of combinations of eye and head movements, as well as head inertia (Keshner and Peterson 1995; Peng et al. 1996). In pigeons, eye movements in response to head fixed motion were found to be directionally accurate but undercompensatory in amplitude (Dickman and Angelaki 1999; Dickman et al. 2000). When the head is free to move, gaze stability was described as being near unity even with passive body restraint (Gioanni 1988). Pigeons involved in natural locomotor behaviors such as standing, perching, walking, and flying were found to maintain a stable head posture orientation that placed the major plane of the horizontal semicircular canals slightly elevated $\left(\sim 5^{\circ}\right)$ relative to the Earth 
horizon (Erichsen et al. 1989). When walking and landing, pigeons exhibit head bobbing behavior, which has been shown repeatedly to contribute to image stabilization (Davies and Green 1988; Frost 1978; Troje and Frost 2000).

Damage to the vestibular system, including hair cell death and denervation produced by ototoxic antibiotics, greatly affects oculomotor, gaze, and postural responses. It has been known for some time that aminoglycoside antibiotics are ototoxic and produce stereocilia loss, edema, hair cell death, and denervation (Berg 1951; Lindeman 1969; Wersäll and Hawkins 1962). With aminoglycoside treatment, functional disabilities immediately result. For example, the important compensatory vestibuloocular reflex (VOR) is diminished or even abolished following administration of ototoxic agents (Carey et al. 1996; Goode et al. 2001; Matsui et al. 2003; Wersäll and Hawkins 1962), as are the vestibulocollic (VCR, Goode et al. 1999) and the visual optokinetic responses (Wersäll and Hawkins 1962). It is now well established that, in time, regeneration of vestibular hair cells, followed by their reinnervation, occurs after the cessation of ototoxic treatments in several animal classes, including birds (Boyle et al. 2001; Dye et al. 1999; Kevetter et al. 2000; Masetto and Correia 1997a, b; Weisleder and Rubel 1992, 1993; Weisleder et al. 1995), amphibians (Baird et al. 1993), and to a lesser extent mammals (Forge et al. 1993; Rubel et al. 1995; Warchol et al. 1993). The morphological regeneration in the vestibular system of birds has been shown to produce some functional recovery of behavioral responses. For example, in chicks compound evoked potentials to linear acceleration stimuli were elicited within a few weeks following ototoxic treatment and had returned to normal after a few months (Jones and Nelson 1992). Carey et al. (1996) reported that the rotational VOR gain to horizontal yaw stimuli recovered coincident with hair cell density increases in the horizontal semicircular canals. Goode et al. (1999) found that the head-stabilizing vestibulocollic reflex (VCR) recovers quickly during regeneration, before either the VOR or significant hair cell populations develop. More recently, Boyle et al. (2001) reported that chick anterior canal afferents lose response sensitivity to rotational motion with ototoxic insult, then recover some functionality after 8 weeks of regeneration where afferent gains remained low and dynamic responses were highly variable compared with normal values. Although these findings are significant, to date no one has examined recovery of posture or head orientation during regeneration in freebehaving pigeons trained to perform specific tasks. It is possible that functional recovery of vestibular-related posture and gaze stability may be accelerated in free-behaving animals, particularly when motivated to meet performance criteria.

In the present study, we produced a complete lesion of all vestibular receptors using ototoxic aminoglycosides that could be documented at a discrete point in time (Frank et al. 1999). We then examined the recovery of vestibular-related head posture and balance associated with vestibular gaze control in pigeons during regeneration.

\section{METHODS}

\section{Animals}

Adult white king pigeons (Columbia livia) were used for this study. Pigeons were chosen because of extensive existing data regarding vestibular function in these animals, as well as their profound regenerative capacity following ototoxic insult. All procedures were performed in accordance with the NIH Guidelines and were approved by the Institution Animal Care and Use Committee.

\section{Testing chamber}

A clear Plexiglas behavioral chamber $(1.75 \mathrm{~m} \mathrm{~L} \times 0.3$ $\mathrm{m} \mathrm{W} \times 0.3 \mathrm{~m} \mathrm{H}$ ) was designed with a separate $0.25-\mathrm{m}^{2}$ holding pen at one end, as shown in Figure 1. The holding pen was isolated by a removable Plexiglas divider. The internal length of the chamber had a 1.5 $\mathrm{m}$ walkway. The bottom of the chamber was lined with corrugated cardboard for traction during movement. One side of the chamber was blackened to prevent distractions. A grid was drawn on top of the chamber to section the run into separate lanes for behavioral quantification. An illuminated pecking key with 3-color display (Lafayette Instruments ENV123A) was mounted at one end, along with a fluid receptacle into which a water reinforcer could be either delivered or removed. Four cameras (Remmington model 14764) were stationed at different angles above and to the sides of the chamber, with all video images recorded simultaneously on four separate tape channels using a JVC video cassette recorder (model HR-S7900U). Behavioral control and reinforcement schedule were adjusted using a microcomputer, a Cambridge Electronic Design interface (model 1401 Plus), and custom scripts written for the Spike II (CED software) environment.

\section{Behavioral training}

Four pigeons were operantly conditioned using a forced-choice modification paradigm. The birds were fluid deprived for $12 \mathrm{~h}$ prior to training and testing. All behavior was videotaped for subsequent offline 


\section{Straight Run Chamber}

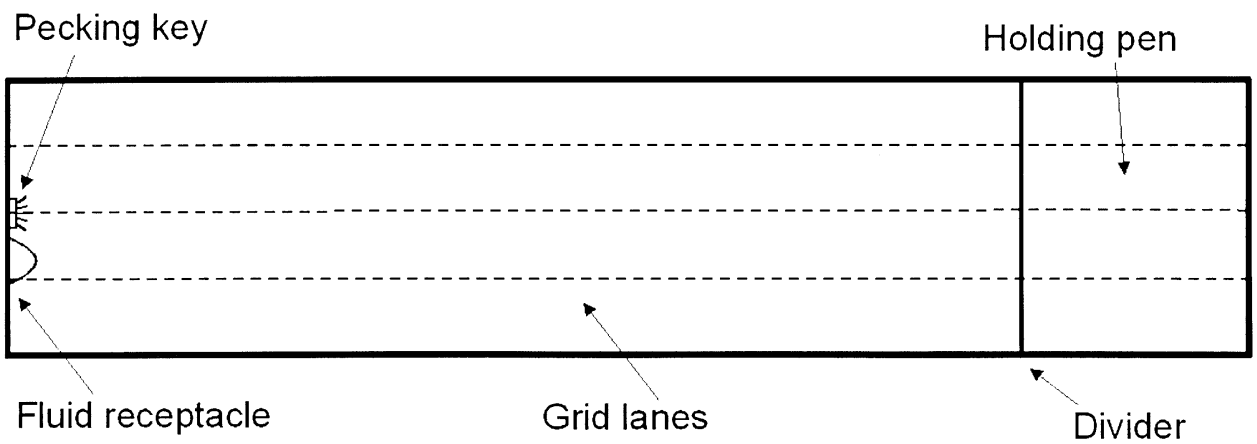

FIG. 1. Straight run behavioral chamber. A $1.75-\mathrm{m}$ behavioral run was used to train the birds using operant conditioning to negotiate along a straight path, peck an illuminated key, and receive water reinforcement. A holding pen was located at one end of the chamber and separated from the path walkway by a Plexiglas divider. Run

analysis. For each trial, the birds were placed in the holding pen. The trial was initiated by illumination of the red pecking key and simultaneous removal of the pen divider. The trial duration consisted of a maximum 30-s interval in which the pigeon must negotiate the 1.5-m run (walk/run from the holding pen to the other end of the chamber), peck the illuminated key, and receive a fluid reward. At the end of each trial, the pigeon would either walk back to the holding pen (or be placed there) and the divider was reset. If the bird did not negotiate the run during the allotted 30-s interval, the trial was scored as a failure and a new trial begun.

\section{Head implant}

Once initial training to the task was complete, a head implant with visual markers was placed on each animal for quantification measures. All surgeries were performed using an aseptic technique. The animal was anesthetized with isoflurane gas $\left(4 \%\right.$ in $\left.\mathrm{O}_{2}\right)$ and intubated. Heart rate was monitored (EKG) and temperature maintained $\left(41^{\circ} \mathrm{C}\right)$ via thermal pad. An incision along the skull midline was made and a small delrin platform was mounted to the skull using three stainless steel screws and dental acrylic. The wound was sutured closed around the implant and the animal was administered buprenex for pain and ampicillin for postoperative infection. The animal was allowed 10 days recovery before behavioral testing resumed.

\section{Behavioral measures}

A white disk with black crossbars was mounted on top of the headpost to serve as a fixed reference point for measuring head position. Normative data was then collected for each pigeon over multiple days with a lanes were marked along the length of the chamber by grid lines. An illuminated pecking key was located at the other end of the chamber, with a fluid receptacle placed adjacent to the key. All trials were computer controlled.

maximum of 10 trials per recorded session. Behavioral measures were scored offline from taped records using frame-by-frame videography (Toshiba W-528). Measurements of head posture and gait were studied. The measures of head posture included gaze saccades, head bobs, and head shakes. Gaze saccades were defined as head movements that consisted of a saccadic motion in one direction that continued to a discrete end point, then terminated with a stationary pause before additional head movement occurred. If the gaze movement consisted of a head saccade that moved, then paused, and moved again in a similar or different direction, a second gaze saccade was counted. Head bobs were defined as a forward head thrust (thrust phase) followed by a period of stationary head in space (hold phase) during which the body moves forward at constant velocity (Frost 1978). Head shake was defined as any high-frequency side-to-side head oscillation that was not exemplary of a head bob or gaze saccade. The variables to assess gait included latency, number of lane changes (path negotiation), and number of steps. Latency was defined as the time between key illumination/divider removal and key peck, or a maximum of $30 \mathrm{~s}$ in the case of failure. Lane changes were defined as the number of times the head center point (black crosshair on head post) crossed any lane marker of the run grid. Due to camera angles, only three lanes drawn along the length of the run (longitudinal) were used. The number of steps was counted from the start of the trial until the key peck, or a maximum of $30 \mathrm{~s}$ in the case of failure. In addition to head gaze and posture, other measures were also quantified. All trials were scored a 1 for a fail, 2 if the bird walked the length of the chamber but did not receive reinforcement, and 3 if there were successful completion of task to receive reinforcement. All measures for all trials were scored twice by the experimenter and averaged. 


\section{Streptomycin}

After normative data was collected, streptomycin (2 $\mathrm{mg}$ ) was surgically delivered bilaterally into the vestibular labyrinths of each bird (Frank et al. 1999). The animal was anesthetized with isoflurane gas $(3 \%$ in $\mathrm{O}_{2}$ ) and intubated. As before, heart rate was monitored and temperature was maintained $\left(40^{\circ} \mathrm{C}\right)$. An incision over the mastoid bone was made and a small bone flap was removed. The vestibule bone was visualized and a small $(0.25 \mathrm{~mm})$ hole was opened into the perilymphatic space near the oval window using a dental burr. A 2-mg streptomycin pellet was inserted into the labyrinth and the opening was closed with a muscle plug and gel foam. The bone flap was replaced and the wound sutured closed. The animal was given buprenex $(0.05 \mathrm{mg} / \mathrm{kg})$ for pain and ampicillin for postoperative infection. During the initial recovery period, hand feeding (Kaytee formula and mixed grains) 3 times a day was administered until independent feeding resumed. Fluid deprivation was not used postoperatively until a minimum of 4 weeks after streptomycin treatment had passed. Recordings were made in intervals of 5 days, 7 days, 14 days, and weekly thereafter through 10 weeks posttreatment. Body weight was monitored daily. Measurements for one bird (2281) were stopped at week 7 due to an acquired, persistent ear infection. An additional bird (2296) did not experience fluid deprivation until week 5 because of sustained low body weight and was continued for experimentation until the 11 week posttreatment time.

\section{Scanning electron microscopy}

In order to assess the extent of damage and the regenerative recovery of the vestibular receptors, scanning electron microscopy was used. A different group of 10 pigeons were administered streptomycin, then allowed to recover for different lengths of time during which regeneration of the receptor cells and reinnervation by vestibular afferents occurred. Each bird was anesthetized (sodium pentobarbital, 250 $\mathrm{mg} / \mathrm{kg}$ ) and the right horizontal and posterior semicircular canals were opened. The labyrinth was perfused with $5 \mathrm{cc}$ of an aldehyde fixative consisting of $2 \%$ glutaraldehyde, $3 \%$ paraformaldehyde, and $1 \%$ acrolein. The animal was then euthanized (1000 mg/ $\mathrm{kg}$ sodium pentobarbital) and the head was removed and placed into the aldehyde fixative overnight. On the following day, the membranous labyrinths were removed from the inner ear and the vestibular receptor epithelia isolated by dissection. Next, all otoconia, otolith membranes, and cupulae were removed and the tissues were placed into a $0.5 \%$ osmium for $2 \mathrm{~h}$. The tissues were washed $(6 \times$ $10 \mathrm{~min}$ ) in distilled water, then dehydrated using a graded series of acetones followed by sublimation with tetramethylsilane (TMS) at $60^{\circ} \mathrm{C}$ (Dye et al. 1999). The tissues were mounted onto studs, palladium coated, and photographed using a Hitachi 2600S scanning electron microscope $(15 \mathrm{kV})$.

\section{Statistics}

Statistical analyses were performed using analyses of variance, with repeated measures when appropriate (ANOVA, Statistica). The measures were compared within animals, where normative data from each bird before streptomycin treatment was used for control.

\section{RESULTS}

\section{Normal behavior}

Four pigeons were trained to manipulate the run and receive fluid reinforcement. As the pecking key was illuminated, the pen divider was lifted and the birds traversed the $1.5 \mathrm{~m}$ run to peck the key and receive the water reinforcement. After remaining for $3 \mathrm{~s}$, the water reward was extracted (by suction) from the receptacle so that satiation could not occur. The bird was then returned to the holding pen for the initiation of a subsequent trial. Each bird was trained to a $100 \%$ success rate for 30 contiguous trials before normative head posture data was obtained. Each trial had to be completed within $30 \mathrm{~s}$ to be considered a success. Following the training procedure, six behavioral measures were observed and quantified as normative data for each bird.

While remaining in the holding chamber prior to trial initiation, the birds would occasionally exhibit some exploratory behavior particularly in the early training phases. Once trained to criterion, however, most often the animal remained directed toward the illumination key until the pen divider was lifted. Normative behavior was characterized by stable upright head posture and little body motion. During the holding period, a few gaze saccades were observed, but no head bobs or head shakes were elicited. At the initiation of the trial by pen divider removal, the trained birds quickly left the holding chamber and traversed the run. For the four birds, the mean latency from key illumination to receipt of reward was $5.5 \mathrm{~s}$ (Table 1). During the walk from the holding pen to the pecking key, the birds exhibited a direct path negotiation strategy that traversed generally along a single lane in the center of the run. Along the path, normal birds elicited an average number of slightly less than 2 lane changes and 11 steps. In addition, during the walking portion of the trial, the 
TABLE 1

Mean values for behavioral measures

\begin{tabular}{lrccrr}
\hline Behavior & Normal & 7 days PST & 28 days PST & 49 days PST & 70 days PST \\
\hline Latency & $6.02(2.6)$ & $30(0.0)$ & $26.1(7.0)$ & $7.2(2.5)$ & $6.6(3.1)$ \\
Lane changes & $2.7(2.3)$ & $20.0(11.7)$ & $18.6(14.3)$ & $3.8(1.0)$ & $1.9(0.8)$ \\
Steps & $10.9(2.9)$ & $17.6(10.0)$ & $30.4(20.5)$ & $13.1(2.5)$ & $11.8(3.2)$ \\
Head bobs & $9.5(3.4)$ & $0(0)$ & $7.1(4.6)$ & $10.1(1.4)$ & $8.3(2.2)$ \\
Head turns & $4.0(3.6)$ & $4.3(8.5)$ & $16.2(4.7)$ & $3.5(3.0)$ & $3.1(2.3)$ \\
Head shakes & $0(0.0)$ & $30.8(16.6)$ & $16.4(22.0)$ & $0.3(0.5)$ & $0 .(0.0)$ \\
\hline
\end{tabular}

Values in parentheses indicate standard deviation.

birds exhibited an average of 10 head bobs, 3 gaze saccades, and zero head shakes (Table 1).

Vestibular receptor cell death and regeneration following streptomycin treatment

Previous studies in our laboratory using identical techniques have shown that a complete receptor cell loss in the epithelia has occurred for all semicircular canal and otolith receptor organs at 4 days time following streptomycin application (Dye et al. 1999; Frank et al. 1999). As a control for the present experiments, the effects of the streptomycin treatment upon the vestibular receptors was assessed in 10 birds at different time points following streptomycin treatments using SEM, as shown in Figure 2. The stereocilia for all hair cells were abolished at 4 days PST, as illustrated by the anterior canal crista epithelium of Figure 2A. Similar to our previous findings with streptomycin-induced lesions (Frank et al. 1999), histologic examination of sectioned material in a separate ongoing investigation regarding vestibular regeneration in pigeons showed that no receptor hair cells were present in any vestibular organ, but support cells remained in the base of the epithelium. Regeneration of the receptor cells was also examined at 14 days PST using SEM, with an increased number of hair cells (as evidenced by stereocilia) present in both the semicircular canals (Fig. 2B) and otolith organs (Fig. 2D). Still, at 14 days PST, the density of receptor cells remained low compared with pretreatment values, particularly in the central regions of the cristae and striola regions of the maculae. These regions are known to contain the majority of the type I hair cells, while the peripheral regions of the epithelia contain exclusively type II cells in birds (Jørgensen and Andersen 1973; Rosenhall 1970; Si et al. 2003; Zakir et al. 2003; personal observations). Counts of cells at 14 days PST (as counted by presence of stereocilia) showed that, in the otolith organs, the density was only $20 \%-25 \%$ of that for normal epithelia (Dye et al. 1999). As vestibular regeneration progressed, the number of hair cells increased. By 42 days PST, the density of receptor cells had reached nearly $50 \%$ of normal values (Fig. 2E). Even at this stage, however, the number of receptor cells in the central region of the cristae and striola region of the maculae were measurably lower than the more peripheral regions of the epithelia. The process of receptor cell generation continued through posttreatment survival (Fig. 2C) and by 12 weeks PST nearly $80 \%$ of the normal cell density had been obtained. In addition, the central region of the cristae and the striola region of the maculae had equilibrated with the level of regeneration seen in the more peripheral locations of the maculae (Fig. 2F).

\section{Behavior following streptomycin treatment}

Initial behavioral deficits. All birds exhibited markedly different behavior initially following the streptomycin treatment. By the second day post-streptomycin treatment (2 days PST), all birds showed pronounced static and locomotor ataxia. Most noticeable was the severe postural instability and lack of head control, as characterized by frequent stumbling, staggering, and an inability to maintain stable positions when standing. Often, the bird would turn or circle in broken oscillations, as illustrated in Figure 3A. The severity of these symptoms progressed over the course of the first 4-7 days PST. The animals also exhibited a severe lack of head stability. There was little observance of normal gaze saccades, instead many rolling or oscillatory head movements were present, with a complete inability to keep the head positioned stationary in space. In fact, the treated birds could no longer maintain the head upright relative to earth vertical (i.e., gravity). Often, violent head thrusts in chaotic directions were observed, particularly when the animal attempted to walk or was circling. These abnormal movements included frequent head shakes, tremors, and prolonged oscillations side to side, as illustrated in Figure 3B.

As a consequence of the head and postural instability, the birds were incapable of orienting in the 

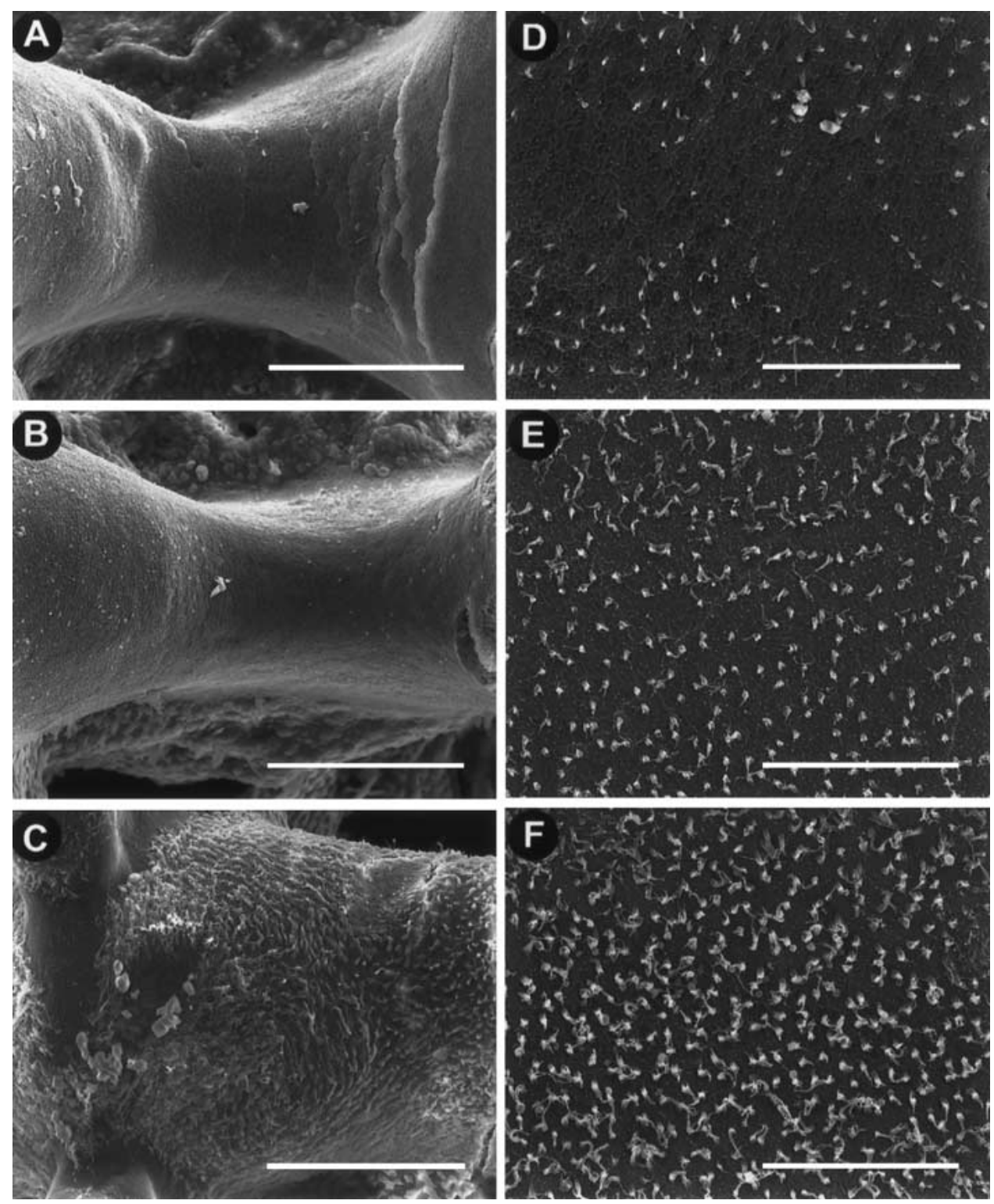

FIG. 2. Scanning electron micrographs of regenerating receptor cells in the semicircular canal crista and utricular macula. A-C. Regeneration in the anterior canal cristae. No stereocilia (receptor cells) are present in the epithelium at 4 days PST (A). Low density of receptors during early regeneration at 14 days PST, with most receptor cells located in the peripheral regions of the epithelium (B). At 63 days PST $(\mathbf{C})$, receptor cell density has increased. All scale bars $=100 \mu \mathrm{m}$. D-F. Regeneration in the utricular maculae. Some early regenerating hair cells are present in the central and peripheral regions of the macula, with few hair cells present in the striola region at 14 days PST (D). By 42 days PST (E), the receptor cell density has increased, but the striola region still contains fewer cells than extrastriola regions. The density of receptor cells has increased for all regions to nearly 80 of normal at 84 days PST $(\mathbf{F})$. Scale bars $=50$ $\mu \mathrm{m}$. behavioral chamber. The first experimental trials were performed at 5 days PST (three birds) and again at 7 days PST (all four birds). When the birds were placed into the holding pen, immediate stumbling, circling, and random directional steps were taken. These deficits were profound, with none of the birds able to negotiate the run. In fact, for all birds, none of the trials was carried to success (100\% failure rate) as all animals remained in the holding pen area and could not orient within the chamber to advance down the run to peck for fluid reinforcement. The three postural and three head gaze behaviors that were quantified all indicated a severe loss of function at 7 days PST, as shown in Table 1. For each bird, the behavioral measures from 10 successive trials were obtained, then averaged together. Failure to perform the task was most apparent in the latency measure, where all trials ended after $30 \mathrm{~s}$ with no successful key peck for any trial or any bird on either day (Table 1). The severity in loss of path negotiation capability was also exhibited in the high number of lane changes, where the bird crossed one of the divider lanes (often while stumbling or circling). For the four birds, the repeated measures within-animal comparisons showed significant increases in number of lane changes. As a group, an average of $20(\mathrm{SD} \pm 11.7)$ lane changes on day 7 PST occurred as a significant increase over the normal number of $2.7(\mathrm{SD} \pm 2.3)$ lane changes $[F(1,78)=52.6, p<0.001]$ before treatment. The postural instability and lack of orientation can be better appreciated when it is noted that all of these increased lane changes occurred either in the holding pen or immediately outside the pen, as no bird moved down the run toward the illuminated key but 


\section{A}

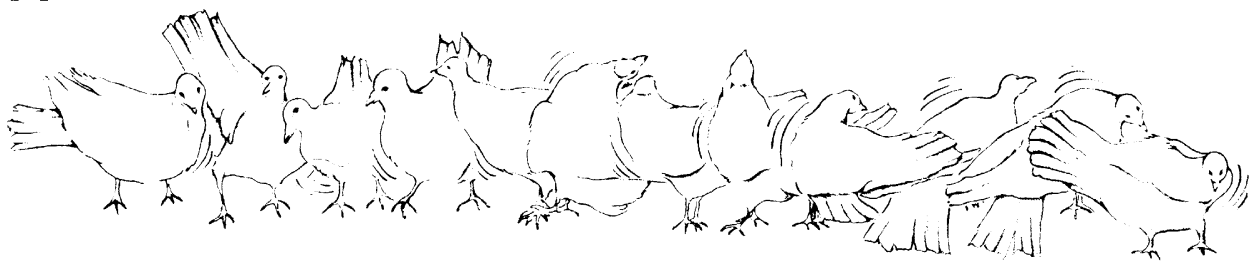

B

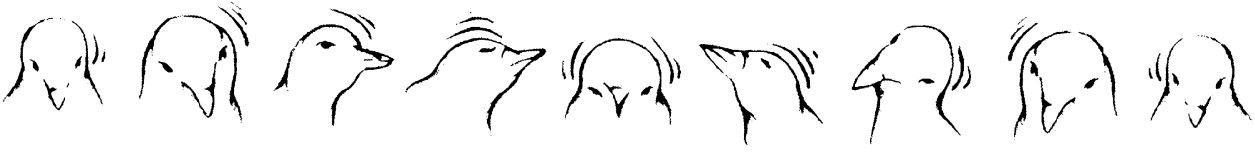

FIG. 3. Circling and head shake behavior of streptomycin-treated birds. A. As illustrated in the cartoon drawings from left to right, early (5-7 days) PST birds exhibited a staggered or broken circling behavior when attempting to stand. The balance posture shifted frequently from leg to leg as the body turned. Head movements were

instead remained near the placement point while staggering or circling. Thus, both the individual within-animal comparisons and the mean number of $17.6(\mathrm{SD} \pm 10)$ steps measured at 7 days PST for all four birds were significantly higher than normal (Table 1), but all occurred as the bird attempted to stand with little directed walking behavior exhibited $[F(1,78)=6.8, p<0.01]$. Head posture was characterized by multiple and frequent tremors and largeamplitude head shakes. The two measures of normal head movements, including gaze saccades and head bobs, were found to be significantly reduced (totally or nearly absent) in all birds (Table 1). For example, the mean of $0(\mathrm{SD} \pm 0)$ head bobs per trial was observed at 7 days PST compared with the normal pretreatment mean of 9.5 (SD \pm 3.4$)$ bobs $[F(1,78)=903.1, p<0.001]$. Instead, head movements consisted of large-amplitude head shakes and high-frequency tremors that were disruptive to orientation. The head shake was characterized by a ballistic head movement (measured to range between 15 and $30 \mathrm{~Hz}$ ) to one side, backward, or in an oblique roll motion. The measured head shakes were significantly more frequent in 7-days-PST birds at an average of $30.8(\mathrm{SD} \pm 16.6)$ shakes per trial compared with none observed in the same birds before streptomycin treatment $[F(1,78)=142.1, p<0.001]$. Head tremors were not quantified for technical reasons; however, they were measured to be $3.0-3.5 \mathrm{~Hz}$ and occurred primarily in the horizontal head plane.

Behavioral recovery during regeneration. The initial head, postural, and orientation dysfunction persisted for several weeks following streptomycin treatment. As shown in Figure 4, the performance measures for postural behaviors, including trial latency, number of steps per trial, and number of lane changes per trial, frequent and chaotic. Many head thrusts were observed in the upward and backward direction, particularly during the turning behavior. B. As illustrated in the cartoon drawings from left to right, PST birds exhibited frequent, rapid side-to-side head shake movements that often contained an upwardly directed component.

were plotted as a function of recovery time following streptomycin treatment. The measures for all 10 trials on each day for each bird were averaged and plotted. As a result of continued kinetic ataxia and apparent lack of orienting capability, all birds were unable to negotiate the run for fluid reinforcement for the first several weeks following streptomycin treatment. The latency values of $30 \mathrm{~s}$ indicate $100 \%$ failure rates for the first three weeks PST (Fig. 4). In fact, the earliest successful trial was observed at 28 days PST by birds 2257 and 2281, with average latencies of $28(\mathrm{SD} \pm 4.1)$ and $15.6(\mathrm{SD} \pm 8.8) \mathrm{s}$, respectively. The remaining two birds followed with successful trials starting at 35 days PST, At this recovery stage, most trials still ended as failures. At the same time, however, gradual improvement in the postural stability increased and each bird began to exhibit some normal behaviors. Thus, the circling behavior for each bird had largely subsided by 3-4 weeks PST, however, some staggering remained and head (gaze) stability was incomplete (see below). By 7 weeks posttreatment, spatial orientation had returned and all birds successfully negotiated the run for 10 consecutive trials to receive fluid reinforcement, with a mean latency value of 7.2 (SD $\pm 2.5) \mathrm{s}$ (Table 1). Only a slight improvement in latency was observed after this point, where the measured values for within-animal comparisons became similar to those obtained before streptomycin treatment was given for each animal (Table 1). The latency measures for each recovery day for individual birds were compared against the pretreatment values. It was found that on the last day of testing for three of the four birds, no significant differences between normal and final latency measures were present. The remaining bird (2257) had a slightly elevated run latency compared with his pretreatment level (Fig. 4). 

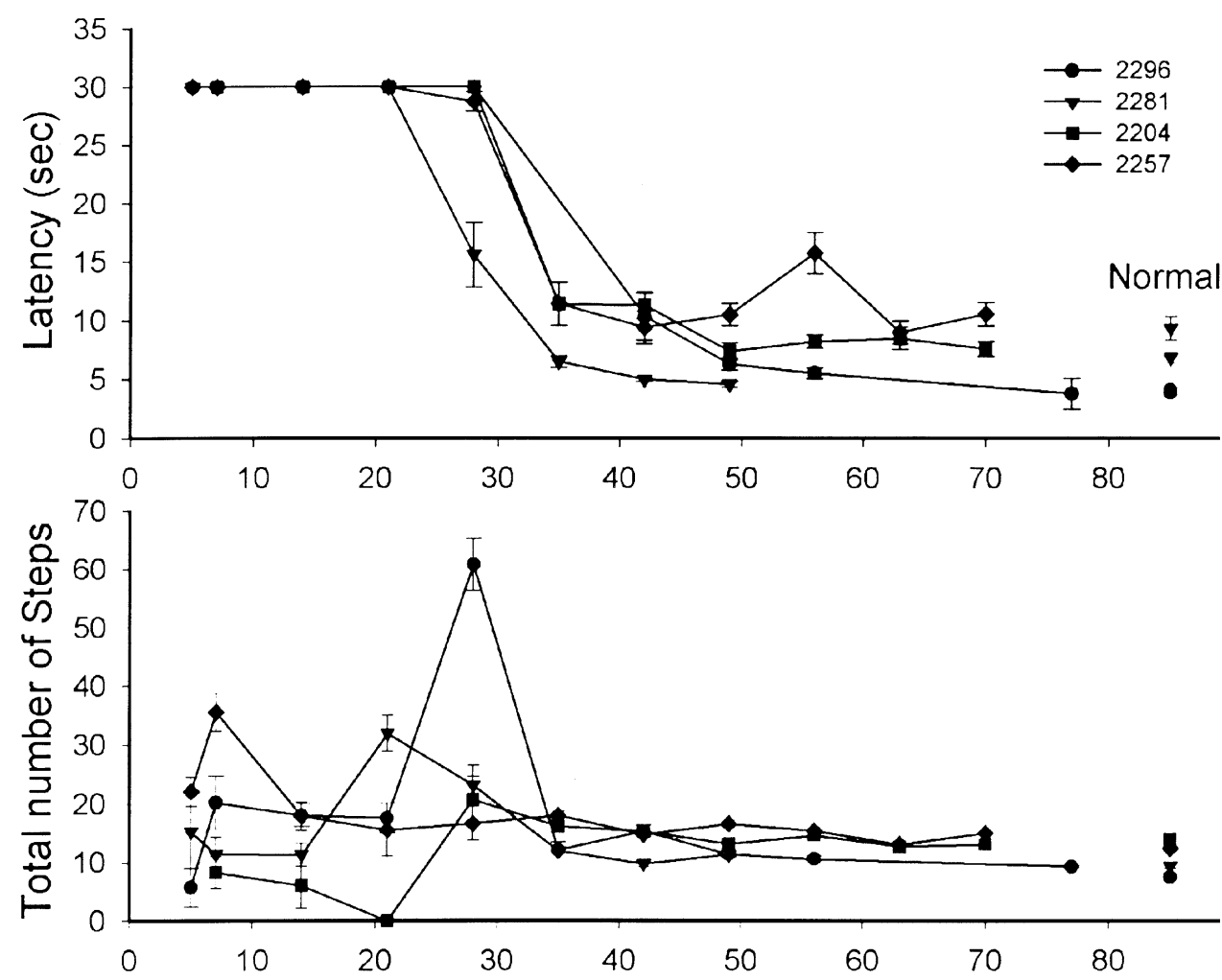

FIG. 4. Latency, number of steps, and number of lane changes as a function of recovery days following streptomycin treatment. The latency (top) was measured as the time between key illumination and key peck when the bird must negotiate the $1.5-\mathrm{m}$ run. The number of steps (middle) was counted during the trial, when directed toward the goal key peck. The lane changes (bottom) were measured as the body crossed one of the three longitudinal lanes from the holding pen to the illuminated key in the run. The averaged responses for 10 trials on each testing day posttreatment are shown for each of four birds. Normal pretreatment values obtained for each bird are shown at the end.

In addition to latency, the degree of ataxia could be assessed by examining the number of lane changes made by the bird as the run was negotiated. When staggering, the animal was unable to pursue a straight course and wobbled from one side of the run to the other, passing through different lanes. All birds exhibited significant large increases in the number of lane changes in the first several weeks following streptomycin treatment (Table 1), which decreased over time to the normal pretreatment levels (Fig. 4). Within-animal comparisons showed that, by 48 days PST, the number of lane changes exhibited during the trial was no different from normal pretreatment levels. In conjunction, there was a wide dispersion in the number of steps taken during trials in the first 3 weeks PST. Initially, many steps were observed during circling and staggering behaviors, but most of these steps were unscored unless they were determined to be directed toward the illuminated pecking key. As vestibular regeneration progressed, postural stability and orienting improved with more directed steps being taken (Fig. 4). Gradually, the birds' walking behavior became spatially oriented, with steps producing movement down the behavioral run toward the illuminated key. By 35 days PST, the increased number of steps had diminished to a constant level for all birds (Fig. 4). At this time in recovery, the mean 13.1 ( $\mathrm{SD} \pm 2.5$ ) steps required to complete the behavioral run and key peck was not significantly different from the mean of 10.9 (SD \pm 2.9 ) steps observed prior to treatment (Table 1). 

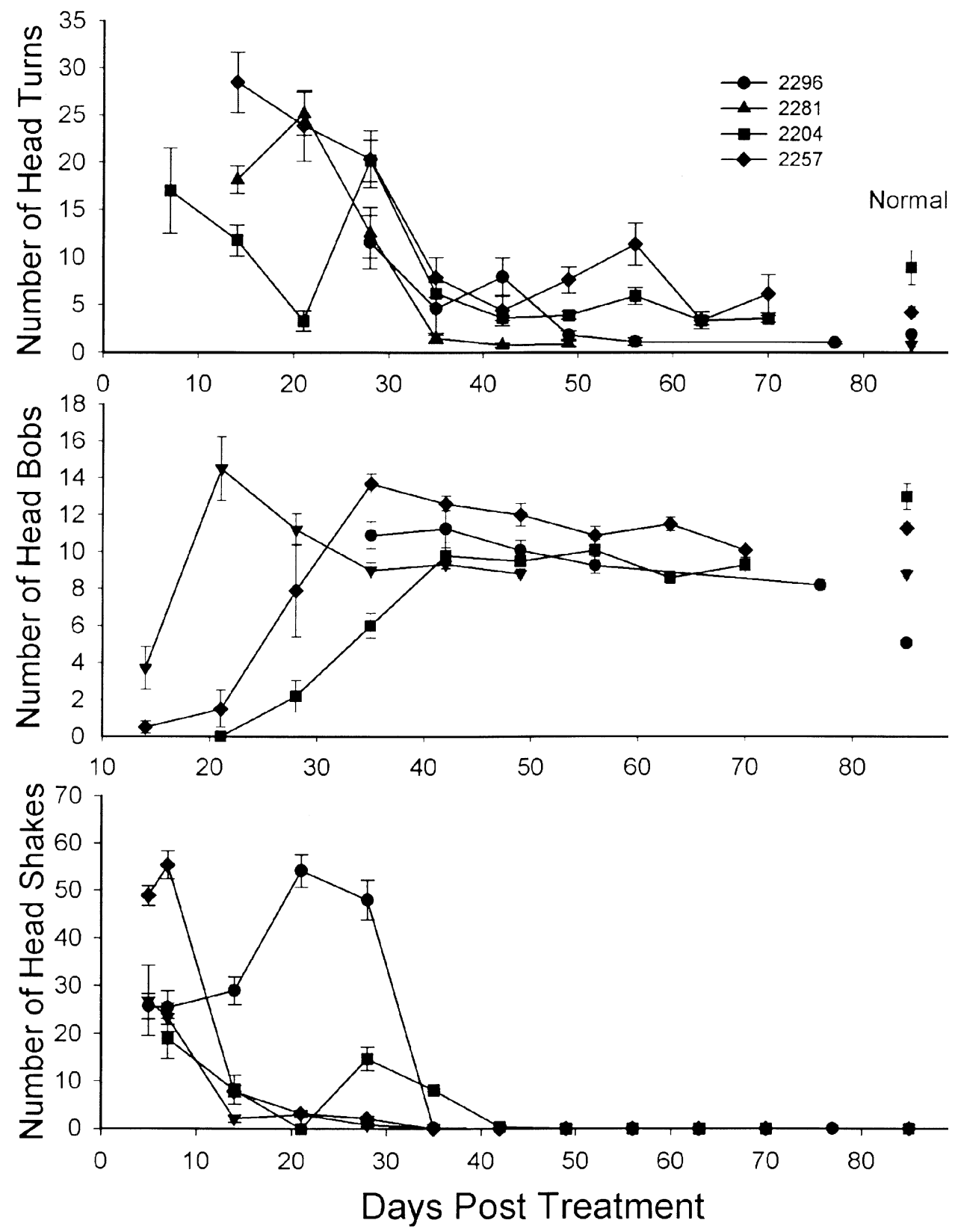

FIG. 5. Number of head gaze saccades, head bobs, and head shakes as a function of recovery days following streptomycin treatment. The number of head gaze saccades (top) was measured when they occurred as a normal saccadic gaze movement. Head bobs (center) are a natural behavioral movement that occurs in pigeons during walking (and other behaviors, but not when standing in one location). Head shakes were observed only in treated birds and could be either side to side or upward and backward movements. The averaged responses for 10 trials on each testing day posttreatment are shown for each of four birds. Normal pretreatment values obtained for each bird are shown at the end.

In addition to posture, the recovery of head stability during regeneration was measured. As noted above, there was an initial loss of normal gaze saccades within 2 days following streptomycin application. As shown in Figure 5, there were few, if any, recognizable gaze saccades in the many head movements observed during the 5 and 7 days PST behavioral trials. Instead, there were frequent, nearly constant, head movements that were best characterized as tremors or head shakes. Two of the four birds presented with these tremors through 4 weeks PST and the other two remaining birds persisted through to 5 weeks. No tremors were observed in data beyond 5 weeks. In addition to the tremor there were quantifiable head shakes that these were measured throughout recovery (Fig. 5). A significantly large increase in the number of head shakes was observed for all birds at 7 days PST (Table 1) with a mean value of 30.8 ( $\mathrm{SD} \pm 16.6$ ) shakes per trial compared with zero during the normal pretreatment control runs $[F(1,78)=142.1, p<0.001)]$. The elevated occurrence of head shakes and lack of gaze saccades persisted for the first two weeks of post-streptomycin recovery. As recovery progressed, the mean number of head shakes gradually diminished while the number of gaze saccades increased (Fig. 5). In fact, by 28 days PST, the number of gaze saccades had significantly increased to values nearly two times normal for three of the four birds, while head shakes had dropped by more than half (Table 1). The fourth bird 
retained head shake behavior with no measurable gaze saccades at 28 days PST. By 49 days PST, the number of head shakes observed during the trials was essentially zero (Table 1), not significantly different from that seen in each animal during pretreatment normative runs. In contrast, the number of gaze saccades had again declined from the large number observed a few weeks earlier to reach normative pretreatment values (Table 1, Fig. 5). The final measure of head posture examined was head bobs. Following the streptomycin application, walking behavior was not possible and the number of head bobs dropped to zero. Three of the four birds exhibited slow increases in the number of elicited head bobs during the first 21 days PST (Fig. 5). In fact, for each bird, head bobs were observed only 1-2 weeks after the appearance of gaze saccades. However, by 28 days PST, the mean number of head bobs had increased greatly (Table 1), although it remained slightly lower than normal due to the more gradual recovery of one bird (Fig. 5). By 49 days PST, there was no significant difference in the mean number of head bobs observed from those obtained during pretreatment trials (Table 1).

\section{DISCUSSION}

\section{Vestibular receptor damage and regeneration}

The present results clearly show that head stability and postural stability in pigeons are greatly dependent upon a functioning vestibular system. A simple learned walking and key peck task was totally eliminated as the motor components necessary for stability and gaze orientation were compromised by vestibular insult. In the present study, direct intralabyrinthine application of streptomycin produced a complete loss of receptor cells and denervation of the semicircular canal and otolith epithelia by four days following treatment, similar to our previous findings in pigeons (Frank et al. 1999). Since the vestibular loss was produced at a discrete point in time, the regenerative capacity of the vestibular system, along with the return of related function, could be directly assessed. Previously, using identical methods, we reported that pigeon vestibular hair cell regeneration followed an exponential growth curve that recovered to normal density values in the maculae by 24 weeks PST (Dye et al. 1999). In addition, the extrastriola regions of the macula and the peripheral regions of the cristae recovered faster with higher cell densities than did the striola region which lagged behind. These extrastriola and peripheral regions in birds have a much higher concentration of type II receptor cells compared with type I cells (Jørgensen and Andersen 1973; Kevetter et al., 2000; Si et al., 2003; Zakir et al.,
2003). Type II cells have repeatedly been shown to regenerate faster than type I cells (Carey et al. 1996; Goode et al. 1999; Kevetter et al. 2000; Masetto and Correia 1997; Weisleder and Rubel 1993).

\section{Initial vestibular dysfunction following streptomycin application}

Within one to two days following the streptomycin application, profound postural and head stability deficits, as well as a loss in spatial orientation were observed in the present study. These substantial behavioral deficits coincide with the complete hair cell loss and afferent denervation produced in these animals. Vestibular neuromotor dysfunction following aminoglycoside administration has also been previously reported for the VOR and VCR (Carey et al. 1996; Goode et al. 1999, 2001; Matsui et al. 2003). However, in these studies using chicks and systemic administration of ototoxic agents, only an intermittent head tremor that persisted for several post treatment days was noted, while no other motor abnormalities were reported (Carey et al. 1996; Goode et al. 1999). In fact, for studying the VCR responses, Goode et al. (1999) required the animals to stand on perches during the rotational motion. The difference in the severity of the postural and head stability deficits observed in our current pigeon study with those in other investigations may lie in several distinctions. First, in the current study a complete hair cell loss and total denervation of the vestibular receptor epithelia occurred (Fig. 1, Dye et al. 1999; Frank et al. 1999). With systemic injections, vestibular receptor cells were greatly diminished but nearly $35 \%-40 \%$ of the semicircular canal cells often remained intact (Carey et al. 1996; Goode et al. 1999; 2001; Matsui et al. 2003). In the utricle, systemic injections typically damaged on even smaller percentage of cells (Lindeman 1969; Matsui et al. 2003; Weisleder and Rubel 1993; Wersäll and Hawkins 1962). Thus, the observed recoveries in chicks were likely not due to regeneration alone but were coupled with possible remaining function of nondamaged hair cells as well as the rescue and repair of sublethally damaged hair cells (Baird et al. 2002). Second, the previous regenerative investigations of VOR and VCR recovery were performed in young hatchling chicks compared with adult pigeons in the current report. It is likely that recovery mechanisms in both the periphery and central compensatory networks work more quickly in younger developing animals than in adults.

\section{Behavioral recovery during regeneration}

In our adult pigeons, recovery from ototoxic insult for the postural and head stability measures followed 
similar time courses. During the first week PST, a striking lack of stability was observed for all animals, where a prevalence of head tremor, head shakes, and circling occurred. These motor behaviors were not normally observed pretreatment and were accompanied by the complete lack of normal gaze saccades, head bobs, and path navigation posttreatment. As observed by the within-animal comparisons, each bird gradually began to exhibit a limited number of directed steps, gaze saccades, and a few head bobs during the second and third weeks of recovery (some interanimal variability was noted for longitudinal recovery time). Still, at this time none of the animals was capable of negotiating the run or performing a key peck. In fact, the birds rarely left the holding pen even when the divider was removed (open field condition) and they exhibited continued staggered movements, many lane changes, and occasional circling. However, during the fourth to fifth weeks of recovery, an interesting change in head stability was noted. The number of head shakes dramatically declined for all birds while the number of recognizable gaze saccades significantly increased. In fact, the gaze saccades increased to a level nearly double that of pretreatment levels. Along with this change came an increase in normal head bobbing behavior.

These results were surprising in that the postural and head stability deficits were not expected to be so severe and last so long. For example, in chicks the VCR was reported to have recovered by 2-3 weeks following systemic administrations of streptomycin, a significantly shorter time course than that observed for head stability in our adult pigeons (Goode et al. 1999). However, in chicks, the total horizontal semicircular canal hair cell density was noted to be near normal values (Goode et al. 1999), although type I hair cells remained reduced in number (Goode et al. 1999; Weisleder and Rubel 1993). In contrast, in pigeons regenerating following intralabyrinthine streptomycin applications, hair cell densities were reported to be only $30 \%$ of normal in the otolith organs (Dye et al. 1999), similar to the qualitative SEM hair cell density counts observed in the present study. In systemically treated regenerating pigeons, hair cell densities of $58 \%$ of normal at 3 weeks PST, nearly $60 \%$ at 49 days PST, and $100 \%$ at 71 days PST were previously reported for the semicircular canals (Kevetter et al., 2000), while the utricular macula had only $75 \%$ of the normal density at the latest stage measured (Kevetter et al. 2000). In terms of function, Massetto and Correia (1997a,b) found that many of the regenerated type II hair cells had some functional ionic currents at 3 weeks PST, although complete physiological maturity was not reached for all type II cells until 9-10 weeks PST. Thus, the slower return of posture and head stability experienced through the first recovery month in our study could be explained by the slower time course of hair cell regeneration compared with chicks. These findings parallel those observed for the recovery of the chick VOR, which returned much later than the VCR response during regeneration (Carey et al. 1996; Goode et al. 2001; Matsui et al. 2003). The recovery of the chick VOR was noted to be correlated with the morphological return of type I but not type II hair cells in the horizontal crista (Carey et al. 1996).

In addition to hair cell regeneration, afferents are also reestablishing innervations in the receptor epithelia and return to providing motion information to central vestibular neurons. Two investigations regarding the physiological responses of regenerating afferents in birds have been reported using systemic ototoxic treatments. In both chicks and pigeons, early (14-18 days) recovery showed that many canal afferents exhibited no response to stimulation, while at 45 weeks PST, more afferents had regained spontaneous rates and response gains that were approximately one quarter to one half the normal level (Boyle et al. 2001; Li and Correia 1998). The recovery of afferent responses in these studies parallels our present observed reduction in head tremor and head shakes at approximately 28 days PST, as well as the increased recovery of gaze saccades and head bobbing. In the present study, by 49 days PST, most measures for postural and head stability, as well as the interanimal variability, had returned to pretreatment normal values. In both chicks and pigeons, as regeneration progressed through 2-5 months following streptomycin application, the afferents showed increased firing rates and had responses with increased gains over a larger stimulus bandwidth. For example, in chicks at 8 weeks posttreatment in some fibers gains were returning to normal values, but many other afferent gains remained abnormally low and their phase relative to head velocity was substantially variant compared with normal birds (Boyle et al. 2001). In pigeons, normal response properties and firing rates were observed for nearly all afferents at 150 days posttreatment ( $\mathrm{Li}$ and Correia 1998). Although with systemic treatments fewer hair cells and afferents are damaged compared with our intralabyrinthine lesions, it would appear that a certain level of afferents must regain near-normal spontaneous firing rates and a head motion sensitivity before central neuronal responses required for providing head and postural stability can minimally function. In fact, many central vestibular neurons that receive direct labyrinthine input have been identified as vestibulospinal projection neurons (Peterson et al. 1980). Some of these vestibulospinal cells also carry eye movement information, suggesting a role in gaze saccades (Boyle 1983; Boyle et al. 1992). Selective stimulation of ei- 
ther the otolith or the semicircular canal nerves has been shown to provide specific activation patterns in different neck muscles subserving head posture, orientation relative to gravity, and rotational head motion (Bolton et al. 1992; Isu et al. 1988; Suguichi et al. 1995; Uchino et al. 1990, 1997; Wilson and Maeda 1974). The functional significance of these activation patterns has not been clearly established; however, their involvement in the recovery of posture, head stability, and gaze control seems likely.

In addition to receptor cell and afferent regeneration, neural plasticity in central vestibular pathways (vestibular compensation) is a constant process that helps stabilize head posture, balance, and vestibular reflex responses (e.g., VOR and VCR) with changes in multisensory inputs to vestibular nuclei networks. For example, with a loss in vestibular afferent input, partial recovery of the VOR and head posture occurs. Central compensation involves visual, proprioceptive, and other extravestibular inputs to vestibular nuclei neurons that develop increased efficacy to alternative sources of motion detection when vestibular afferent inputs decrease (Precht et al. 1981; Lacour and Xerri 1981; Zennou-Azogui et al. 1996). For example, with unilateral vestibular loss, compensation in the central neuron responses and the VOR for slow head movements occurs rapidly but fast head movement responses recover much more slowly (months to years), with some deficits lasting for the life of the animal (Baarsma and Collewijn 1975; Dieringer 1988; Fetter and Zee 1988; Halmagyi et al. 1990; Newlands and Perachio 1990). In the present study, central compensation surely occurred with the bilateral loss of vestibular afferent drive. In addition, as new receptors regenerated and afferents began to reinnervate the epithelia, neural plasticity would be expected to continue at the central level as motion signals returned. The mechanisms of recovery in the central pathways underlying the regenerative recovery in posture, head stability, and navigational behavior observed should provide interesting study and remain the subject of future investigations.

In the present study, recovery of the orientation and navigational cues necessary to perform trained behavior was more delayed relative to the recovery of postural and head stabilities. For example, all birds completely failed to negotiate the run and key peck for reinforcement for the first three weeks following the streptomycin treatment. In fact, it was not until the fourth or fifth week PST that any successful trial was performed for any bird, and even at 5 weeks PST, most trials resulted in failure. This was true even though head stability had significantly increased and the ataxia had largely subsided, as best noted by examining withinanimal comparisons. These observations, coupled with the continued occurrence of misdirected steps, in- creased lane changes, and a substantially increased number of gaze saccades, all suggest that the birds could not spatially orient well in the chamber. Prior to the point where head shakes became directed gaze saccades, orientation remained difficult for each of the pigeons as evidenced by the occurrence of a large number of lane changes and longer latencies (failed trials, within-animal comparison). Difficulties in spatial orientation have been previously reported with vestibular deficits. For example, head direction cells in the hippocampus and anterior thalamus exhibit preferred directions for specific head orientations in space (Knierim et al. 1998; Taube 1995, 1998; Taube et al. 1990). Through distributed networks these neurons are thought to provide the current directional heading of the animal, an essential construct for spatial orientation (Zhang 1996; Zugaro et al. 2003). Head direction cells have been shown to require vestibular inputs for acquisition of directional selectivity (Stackman and Taube 1997; Stackman etal. 2002), and vestibular nerve lesions have also been reported to affect navigational ability (Stackman and Herbert 2002). Whether the loss of vestibular signals from our ototoxic lesions compromised the ability of the birds to negotiate the run through a loss in navigational ability is unknown, but it would be consistent with the present findings. It is interesting to note that only after substantial head stabilization and postural control returned were the birds capable of negotiating the run and producing a key peck. It is possible that muscle atrophy occurred during the prolonged ataxic period, which significantly contributed to the reduced navigational ability. However, if only a motor deficit were the result of the vestibular lesion, one would predict that the latency measures would be reduced and the number of successful trial occurrences would be higher at approximately 21 or 28 days PST, instead of one or two weeks later. It also clear from these results that all birds retained the capacity to perform the behavioral task without relearning once the postural and head stability neuromotor function had been reacquired. Thus, the loss of vestibular receptor cells and afferent denervation through ototoxicity, although undoubtedly affecting central vestibular neuronal responses to motion, did not result in a loss of learned task behavior. Instead, the present study suggests that vestibular dysfunction not only produces motor deficits in postural and head stability but may also severely affect spatial orientation and navigation that require longer recovery periods.

\section{ACKNOWLEDGMENTS}

The authors would like to thank David Huss for his excellent support and contributions. The authors would also like to thank Erin and Jennifer White for their superb animal 
care. The work was supported in part by grants from NIH (DC03286) and NASA (NCC2-1159).

\section{REFERENCES}

BaArsma EA, Collewijn H. Changes in compensatory eye movements after unilateral labyrinthectomy in the rabbit. Arch. Otol. 211:219-230, 1975.

BAIRd RA, Torres MA, Schuff NR. Hair cell regeneration in the bullfrog vestibular otolith organs following aminoglycoside toxicity. Hear. Res. 65:164-174, 1993.

Baird RA, Burton MD, Fashena DS, Naeger RA. Hair cell recovery in mitotically blocked cultures of the bullfrog saccule. Proc. Natl. Acad. Sci. USA 97:11722-11729, 2002.

Berg K. Toxic effect of streptomycin on vestibular and cochlear apparatus experimental study on cats. Acta Otolaryngol. Suppl. 97:1.

Bolton PS, Endo K, Goto T, Imagawa M, Sasaki M, Uchino Y, Wilson VJ. Connections between utricular nerve and dorsal neck motoneurons of the decerebrate cat. J. Neurophysiol. 67:16951697, 1992.

Boyle R. Activity of medial vestibulospinal tract cells during rotation and ocular movement in the alert squirrel monkey. J. Neurophysiol. 70:2176-2180, 1983.

Boyle R, Goldberg JM, Highstein SM. Inputs from regularly and irregularly discharging vestibular nerve afferents to secondary neurons in squirrel monkey vestibular nuclei. III. Correlation with vestibulospinal and vestibuloocular output pathways. J. Neurophysiol. 68:471-484, 1992.

Boyle R, Highstein SM, Carey JP, Xu J. Functional recovery of anterior semicircular canal afferents following hair cell regeneration in birds. J. Assoc. Res. Otolaryngol 3:149-166, 2001.

Carey JP, Fuchs AF, Rubel EW. Hair cell regeneration and recovery of the vestibulo-ocular reflex in the avian vestibular system. J. Neurophysiol. 76:3301-3312, 1996.

Crane BT, Demer JL. Human gaze stabilization during natural activities: Translation, rotation, magnification and target distance effects. J. Neurophysiol. 78:2129-2144, 1997.

Davies MNO, GREEN PR. Head-bobbing during walking, running and flying: Relative motion perception in the pigeon. J. Exp. Biol. 138:71-91, 1988.

Dickman JD, Angelaki DE. Three-dimensional organization of vestibular-related eye movements during off-vertical axis rotation and linear translation in pigeons. Exp. Brain. Res. 129:391-400, 1999.

DickMAn JD, Beyer M, Hess BJM. Three-dimensional organization of vestibular related eye movements during rotational motion in pigeons. Vision Res. 40:2831-2844, 2000.

DIERINGER N. Immediate saccadic substitution for deficits in dynamic vestibular reflexes of frogs with selective peripheral lesions. Prog. Brain Res. 76:403-409, 2000.

Dye BJ, Frank T, Newlands SD, Dickman JD. Distribution and time course of hair cell regeneration in the pigeon utricle. Hear. Res. 133:17-26, 1999.

Erichsen JT, Hodos W, Evinger C, Bessette BB, Phillips SJ. Head orientation in pigeons: postural, locomotor, and visual determinants. Brain Behav. Evol. 33:268-278, 1989.

Fetter M, Zee DS. Recovery from unilateral labyrinthectomy in rhesus monkey. J. Neurophysiol. 59:370-393, 1988.

Forge A, Lie L, Corwin JT, Nevill G. Ultrastructural evidence for hair cell regeneration in the mammalian inner ear. Science 259:1616-1619, 1993

Frank TC, Dye BJ, Newlands SD, Dickman JD. Streptomycin ototoxicity and hair cell regeneration in the pigeon utricle. Laryngoscope 109:356-361, 1999.
Frost BJ. The optokinetic basis of head-bobbing in the pigeon. J. Exp. Biol. 74:187-195, 1978.

Gioanni H. Stabilizing gaze reflexes in the pigeon (Columba livia) II. Vestibulo-ocular (VOR) and vestibulo-collic (closed-loop VCR) reflexes. Exp. Brain Res. 69:583-593, 1988.

Goode CT, Carey JP, Fuchs AF, Rubel EW. Recovery of the vestibulocolic reflex after aminoglycoside ototoxicity in domestic chickens. J. Neurophysiol. 81:1025-1035, 1999.

Goode CT, Maney DL, Rubel EW, Fuchs AF. Visual influences on the development and recovery of the vestibuloocular reflex in the chicken. J. Neurophysiol. 85:1119-1128, 2001.

Halmagy GM, Curthoys IS, Cremer PD, Henderson CJ, Todd MJ, Staples MJ, D'Cruz DM. The human horizontal vestibulo-ocular reflex in response to high-acceleration stimulation before and after unilateral vestibular neurectomy. Exp. Brain Res. 81:479_ 490, 1990.

Isu N, Uchino Y, Nakashima H, Satoh S, Ichikawa T, Watanabe S. Axonal trajectories of posterior canal-activated secondary vestibular neurons and their coactivation of extraocular and neck flexor motoneurons in the cat. Exp. Brain Res. 70:181-191, 1988.

Jones TA, NELSON RC. Recovery of vestibular function following hair cell destruction by streptomycin. Hear. Res 62:181-186, 1992.

Jørgensen JM, ANDERSEn T. On the structure of the avian maculae. Acta Zool 54:121-130, 1973.

Keshner EA, Peterson BW. Mechanisms controlling human head stabilization. I. Head-neck dynamics during random rotations in the horizontal plane. J. Neurophysiol. 73:2293-2301, 1995.

Kevetter GA, Blumberg KR, Correia MJ Hair cell and supporting cell density and distribution in the normal and regenerating posterior crista ampullaris of the pigeon. Int. J. Dev. Neurosci. 18:855-867, 2000.

KNIERIM JJ. Kudrimoti H, McNaughton BL. Interaction between idiothetic cues and external landmarks in the control of place cells and head direction cells. J. Neurosci. 18:425-446, 1998.

LACOUR M, XERRI C. Compensation of postural reactions to fall in the vestibular neurectomized monkey. Role of the visual motion cues. Exp. Brain Res. 40:103-110, 1980.

Li W, CORREIA MJ. Recovery of semicircular canal primary afferent activity in the pigeon after streptomycin ototoxicity. J. Neurphysiol. 80:3297-3311, 1998.

LindEMAN HH. Studies on the morphology of the sensory regions of the vestibular apparatus. Ergeb. Anat. Entwicklungsgesch. 42:1113,1969

Masetto S, Correia MJ. Ionic currents in regenerating avian vestibular hair cells. Int. J. Dev. Neurosci. 15:387-399, 1997a.

MasetTo S, Correia MJ. Electrophysiological properties of vestibular sensory and supporting cells in the labyrinth slice before and during regeneration. J. Neurophysiol. 78:1913-1927, 1997b.

Matsui Ji, Haque A, Huss D, Messana EP, Alosi JA, Roberson DW, Cotanche DA, Dickman JD, Warchol ME. Caspase inhibitors promote vestibular hair cell survival and function after aminoglycoside treatment in vivo. J. Neurosci. 23:6111-6122, 2003.

Newlands SD, Perachio AA. Compensation of horizontal canal related activity in the medial vestibular nucleus following unilateral labyrinth ablation in the decerebrate gerbil. Exp. Brain Res. 82:359-372, 1990.

Peng GCY, Hain TC, Peterson BW. A dynamical model for reflex activated head movements in the horizontal plane. Biol. Cybern. 75:309-319, 1996.

Peterson BW, Fukushima K, Hirai N, Schor RH, Wilson VJ. Responses of vestibulospinal and reticulospinal neurons to sinusoidal vestibular stimulation. J. Neurophysiol. 43:1236-1250, 1980.

Precht W, Maioli C, Dieringer N, Cochran S. Mechanisms of compensation of the vestibule-ocular reflex after vestibular neurotomy. In: Flohr H, Precht W (eds) Lesion-induced Neu- 
ronal Plasticity in Sensorimotor Systems. Springer-Verlag, Berlin, pp 222-230, 1981.

Rosenhall U. Some morphological principles of the vestibular maculae in birds. Arch. Klin. Exp. Ohren. Nasen. Kehlkopfheilkd 197:154-182, 1970.

Rubel EW, Dew LA, Roberson DW. Mammalian vestibular hair cell regeneration. Science 267:701-703, 1995.

Si X, ZaKiR M, DickMan JD Afferent innervation of the uticular macula in pigeons. J. Neurophysiol. 89:1660-1677, 2003.

Stackman RW, Herbert AM. Rats with lesions of the vestibular system require a visual landmark for spatial navigation. Behav. Brain Res. 128:27-40, 2002.

Stackman RW, Taube JS. Firing properties of head direction cells in rat anterior thalamic neurons: Dependence upon vestibular input. J. Neurosci. 17:4349-4358, 1997.

Stackman RW, Clark AS, Taube JS. Hippocampal spatial representations require vestibular input. Hippocampus 12:291-303, 2002.

Sugiuchi Y, Izawa Y, Shinoda Y. Trisynaptic inhibition from the contralateral vertical semicircular canal nerves to neck motoneurons mediated by spinal commissural neuron. J. Neurophysiol. 73:1973-1987, 1995.

TAUBE JS. Head direction cells recorded in the anterior thalamic nuclei of freely moving rats. J. Neurosci. 15:70-86, 1995.

TAube JS. Head direction cells and the neurophysiological basis for a sense of direction. Prog. Nurobiol. 55:1-32, 1998.

Taube JS, Muller RU, Ranck JB Jr. Ranck Head-direction cells recorded from the postsubiculum in freely moving rats. Description and quantitative analysis. J. Neurosci. 10:420-435, 1990.

Troje NF, Frost BJ. Head-bobbing in pigeons: How stable is the hold phase? J. Exp. Biol. 203:935-940, 2000.

Uchino Y, Isu N, Sakuma A, Ichikawa T, Hiranuma K. Axonal trajectories of inhibitory vestibulocollic neurons activated by the anterior semicircular canal nerve and their synaptic effects on neck motoneurons in the cat. Exp. Brain Res. 82:14-24, 1990.

Uchino Y, Sato H, Sasaki M, Imagawa M, Ikegami H, Isu N, Graf W. Sacculocollic reflex arcs in cats. J. Neurophysiol. 77:3003-3012, 1997.

Warchol ME, Lambert PR, Goldstein BJ, Forge A, Corwin JT. Regenerative proliferation in inner ear sensory epithelia from adult guinea pigs and humans. Science 259:1619-1622, 1993.

WeIsleder P, Rubel EW. Hair cell regeneration in the avian vestibular epithelium. Exp. Neurol. 115:2-6, 1992.

WeIsLEDER P, Rubel EW. Hair cell regeneration after streptomycin toxicity in the avian vestibular epithelium. J. Comp. Neurol. 331:97-110, 1993.

Weisleder P, Tsue TT, Rubel EW. Hair cell replacement in avian vestibular epithelium: Supporting cell to Type I hair cell. Hear. Res. 82:125-133, 1995.

Wersäll J, HaWKINS JE Jr. The vestibular sensory epithelia in the cat labyrinth and their reactions in chronic streptomycin intoxication. Acta Otol. 54:1-23, 1962.

WiLSON VJ, MAEDA M. Connections between semicircular canals and neck motoneurons in the cat. J. Neurophysiol. 37:346-357, 1974.

ZAKIR M, Huss D, DickMAn JD Afferent innervation patterns of the saccule in pigeons. J. Neurophysiol. 89:534-550, 2003.

Zennou-Azogui Y, Xerri C, Leonard J, Tighilet B. Vestibular compensation: role of visual motion cues in the recovery of posturokinetic functions in the cat. Behav. Brain Res. 74:65-77, 1996.

ZHANG K. Representation of spatial orientation by the intrinsic dynamics of the head-direction ensemble: a theory. J. Neurosci. 16:2112-2126, 1996.

Zugaro MB, Arleo A, Berthoz A, Wiener SI. Rapid spatial reorientation and head direction cells. J. Neurosci. 23:3478-3482, 2003. 